\title{
Stark broadening of neutral zinc spectral lines
}

\author{
M.S. Dimitrijević ${ }^{1}$ and S. Sahal-Bréchot ${ }^{2}$ \\ 1 Astronomical Observatory, Volgina 7, 11000 Belgrade, Serbia, Yugoslavia \\ ${ }^{2}$ Laboratoire "Astrophysique, Atomes et Molécules", Département Atomes et Molécules en Astrophysique, \\ Unité associée au CNRS 812, Observatoire de Paris-Meudon, 92190 Meudon, France
}

Received June 24; accepted September 6, 1999

\begin{abstract}
Using the semiclassical perturbation approach, we have calculated electron-, proton- and He II-impact line widths and shifts for $32 \mathrm{Zn}$ I multiplets as a function of temperature and perturber density. Obtained results have been compared with the existing theoretical and experimental data.
\end{abstract}

Key words: atomic data - line: profiles

\section{Introduction}

Neutral zinc spectral lines are present in stellar (see e.g. Sneden et al. 1991) and solar (see e.g. Biémont \& Godefroid 1980; Grevesse 1984) spectra. Consequently, Stark broadening parameters of Zn I lines are of interest for a number of astrophysical problems, e.g. for abundance determinations, as well as for stellar plasma analysis, modeling and diagnostics. Such data are also of interest for laboratory plasma diagnostics, modeling and investigation (see Grechikhin 1969; Kusch \& Oberschelp 1967; Salakhov 1975; Fishman et al. 1979; Dimitrijević \& Konjević 1983; Lakićević 1983; Rathore et al. 1985; Rao et al. 1989).

Within the semiclassical-perturbation formalism (Sahal-Bréchot 1969a,b; see also Sahal-Bréchot 1974; Dimitrijević \& Sahal-Bréchot 1984), we have calculated electron-, proton-, and ionized helium-impact line widths and shifts for 32 neutral zinc multiplets, as the continuation of our project to create a large Stark broadening data set for astrophysical and laboratory plasma research purposes.

Send offprint requests to: M.S. Dimitrijević

e-mail: mdimitrijevic@aob.bg.ac.yu

*Table 1 is only available in electronic form: The material published electronically can be accessed: by ftp at cdsarc.u-strasbg.fr (130.79.128.5) or via http://cdsweb.ustrasbg.fr/Abstract.html

\section{Results and discussion}

In order to determine the full line width at half maximum $W$ and the line shift - $d$ of neutral calcium lines influenced by the Stark broadening mechanism, the semiclassical perturbation formalism has been used. This formalism, as well as the corresponding computer code (Sahal-Bréchot 1969a,b), have been updated and optimized several times (Sahal-Bréchot 1974; Fleurier et al. 1977; Dimitrijević \& Sahal-Bréchot 1984; Dimitrijević et al. 1991; Dimitrijević \& Sahal-Bréchot 1996). A review of the calculation procedure, with the discussion of updatings and validity criteria, is published, e.g., in Dimitrijević (1996, 1997).

Atomic energy levels needed for calculations have been taken from Sugar \& Musgrove (1995). The oscillator strengths have been calculated within the Coulomb approximation (Bates \& Damgaard 1949, and the tables of Oertel \& Shomo 1968). For higher levels, the method of Van Regemorter et al. (1979) has been used.

Electron-, proton-, and He II-impact broadening parameters for $\mathrm{Zn}$ I for perturber densities of $10^{13} \mathrm{~cm}^{-3}-10^{19} \mathrm{~cm}^{-3}$ and temperatures from $2500 \mathrm{up}$ to $50000 \mathrm{~K}$, are presented in Table 1 (accessible only in electronic form). For perturber density of $10^{13} \mathrm{~cm}^{-3}$, only data for three multiplets are shown, since other data are linear with density for densities lower than $10^{14} \mathrm{~cm}^{-3}$. For perturber densities lower than $10^{13} \mathrm{~cm}^{-3}$, Stark broadening parameters for all tabulated multiplets are linear with perturber density. We also specify a parameter $C$ (Dimitrijević \& Sahal-Bréchot 1984), which gives an estimate for the maximum perturber density for which the line may be treated as isolated when it is divided by the corresponding full width at half maximum. For each value given in Table 1 , the collision volume $(V)$ multiplied by the perturber density $(N)$ is much less than one and the impact approximation is valid (Sahal-Bréchot 1969a,b). Values for $N V>0.5$ are not given and values for $0.1<N V \leq 0.5$ are denoted by an asterisk. Stark broadening parameters for densities lower than tabulated are linear with perturber density. 
Table 2. Comparison between experimental and theoretical Stark full widths at half maximum. Experimental data: a-Kusch \& Oberschelp (1967); b-Fishman et al. (1979) c-Rathore et al. (1985). Theoretical data: WDSB - present results; WDK - Dimitrijević \& Konjević (1983)

\begin{tabular}{|c|c|c|c|c|c|c|c|}
\hline Transition & $\begin{array}{l}\text { Wave length } \\
\text { (A) }\end{array}$ & $T(K)$ & $\begin{array}{c}N / 10(+17) \\
(\mathrm{cm}-3)\end{array}$ & Wm & Wm/WDSB & Wm/WDK & $R$ \\
\hline \multirow[t]{14}{*}{$2 n 14 p-5 s$ (triplets) } & 4810.53 & 11000 & 1.0 & 1.65 & 3.56 & 3.25 & $a$ \\
\hline & & 11000 & 4.5 & 0.92 & 0.43 & 0.40 & \\
\hline & & 13700 & 1.0 & 1.00 & 2.24 & 2.02 & c \\
\hline & & 14800 & 1.0 & 0.90 & 1.99 & 1.79 & $=$ \\
\hline & & 16300 & 1.0 & 0.52 & 1.13 & 1.01 & $c$ \\
\hline & & 18100 & 1.0 & 0.40 & 0.86 & 0.76 & c \\
\hline & 4722.16 & 11000 & 1.0 & 1.57 & 3.39 & 3.09 & a \\
\hline & & 11000 & 4.5 & 1.20 & 0.58 & 0.52 & \\
\hline & & 13700 & 1.0 & 1.00 & 2.24 & 2.02 & $c$ \\
\hline & & 14800 & 1.0 & 0.64 & 1.42 & 1.27 & c \\
\hline & & 16300 & 1.0 & 0.42 & 0.92 & 0.82 & 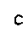 \\
\hline & & 18100 & 1.0 & 0.36 & 0.77 & 0.69 & c \\
\hline & 4680.14 & 11000 & 1.0 & 0.84 & 1.81 & 1.66 & a \\
\hline & & 11000 & 4.5 & 1.29 & 0.62 & 0.56 & $b$ \\
\hline \multirow[t]{3}{*}{$4 p-6 s$ (triplets) } & 3072.06 & 11000 & 1.0 & 0.70 & 0.76 & 0.65 & a \\
\hline & 3035.78 & 11000 & 1.0 & 0.61 & 0.66 & 0.57 & a \\
\hline & 3018.36 & 11000 & 1.0 & 0.56 & 0.61 & 0.52 & a \\
\hline \multirow[t]{3}{*}{$4 p-4 d$ (triplets) } & 3345.02 & 11000 & 1.0 & 1.74 & 2.87 & 2.66 & a \\
\hline & 3302.58 & 11000 & 1.0 & 1.40 & 2.31 & 2.14 & a \\
\hline & 3282.33 & 11000 & 1.0 & 0.91 & 1.50 & 1.39 & a \\
\hline \multirow[t]{3}{*}{$4 p-5 d$ (triplets) } & 2800.9 & 11000 & 1.0 & 1.96 & 0.53 & 0.39 & \\
\hline & 2770.9 & 11000 & 1.0 & 2.49 & 0.67 & 0.50 & \\
\hline & 2756.45 & 11000 & 1.0 & 1.27 & 0.34 & 0.25 & \\
\hline
\end{tabular}

When the impact approximation is not valid, the ion broadening contribution may be estimated by using the quasistatic approach (Sahal-Bréchot 1991 or Griem 1974). In the region between where neither of these two approximations is valid, a unified type theory should be used. For example in Barnard et al. (1974), simple analytical formulas for such a case are given. The accuracy of the results obtained decreases when broadening by ion interactions becomes important.

There are three experimental studies with data of Stark widths and shifts of neutral zinc lines (Kusch \& Oberschelp 1987; Fishman et al. 1979; Rathore et al. 1985). Theoretical data suitable for comparison with our results are published in Dimitrijević \& Konjević (1983), Lakićević (1983), and Rathore et al. (1985).

In Tables 2 and 3, experimental (Kusch \& Oberschelp 1987; Fishman et al. 1979; Rathore et al. 1985) Stark widths (Table 2) and shifts (Table 3) are compared with present results and with semiclassical Stark broadening parameters from Dimitrijević \& Konjević (1983). In the experiments of Kusch \& Oberschelp (1987) and Fishman et al. (1979), ion perturbers are protons, while in the experiment of Rathore et al. (1985) the carrier gas was neon. In order to make the adequate comparison, Stark broadening of neutral zinc by impacts with neon ions has been calculated and included in WDSB and dDSB (present
Table 3. Comparison between experimental and theoretical Stark shifts. Experimental data: c-Rathore et al. (1985). Theoretical data: dDSB - present results; dDK - Dimitrijević \& Konjević (1983)

\begin{tabular}{|c|c|c|c|c|c|c|c|}
\hline Transition & $\begin{array}{l}\text { Wave length } \\
\text { (A) }\end{array}$ & $T(K)$ & $\begin{array}{c}\mathrm{N} / 10(+17) \\
(\mathrm{cm}-3)\end{array}$ & $\begin{array}{l}d m \\
\text { (A) }\end{array}$ & $\mathrm{dm} / \mathrm{dDSB}$ & $d m / d D K$ & Ref. \\
\hline \multirow[t]{9}{*}{ 2n I $4 p-5 s$ (triplets) } & 4810.53 & 13700 & 1.0 & 0.44 & 1.24 & 1.58 & c \\
\hline & & 14800 & 1.0 & 0.35 & 0.97 & 1.24 & c \\
\hline & & 16300 & 1.0 & 0.25 & 0.66 & 0.87 & c \\
\hline & & 18100 & 1.0 & 0.21 & 0.55 & 0.72 & c \\
\hline & 4722.16 & 11000 & 4.5 & 0.78 & 0.49 & 0.69 & b \\
\hline & & 13700 & 1.0 & 0.44 & 1.24 & 1.58 & $c$ \\
\hline & & 14800 & 1.0 & 0.35 & 0.97 & 1.24 & c \\
\hline & & 16300 & 1.0 & 0.256 & 0.67 & 0.89 & $c$ \\
\hline & & 18100 & 1.0 & 0.175 & 0.46 & 0.60 & c \\
\hline
\end{tabular}

calculation of Stark widths - WDSB and shifts - dDSB) values in Tables 2 and 3 . For the $4 \mathrm{p}^{3} \mathrm{P}^{\circ}-5 \mathrm{~d}^{3} \mathrm{D}$ multiplet, the impact approximation is not valid for proton perturbers and the quasistatic ion broadening contribution is calculated according to Griem (1974). One can see in Table 2 that the agreement of all experiments with both calculations is very poor. The ratio of experimental widths of Kusch \& Oberschelp (1967) and the theoretical ones vary from 0.25 up to 3.56 . The experimental widths of Fishman et al. (1979) are two times larger than theoretical values from both approaches. The temperature trend of the experimental widths of Rathore et al. (1985) is in such disagreement with both theoretical approaches that the ratios of measured and calculated Stark widths vary e.g. for the $4722.16 \AA$ line from 2.24 for $T=13700 \mathrm{~K}$ up to 0.77 for $T=18100 \mathrm{~K}$ for the present results, and from 2.02 up to 0.69 for the theoretical values of Dimitrijević \& Konjević (1983). For the shift, ratios of experimental values of Rathore et al. (1985) and results of the present calculations vary from 1.24 to 0.46 for the same spectral line.

The experimental results of Fishman et al. (1979) and Rathore et al. (1985) were not selected for critical compilations of reliable Stark broadening experimental data (Konjević \& Roberts 1976; Konjević et al. 1984; Konjević \& Wiese 1990), while the results of Kusch \& Oberschelp (1967) were selected with the attribution of the lowest accuracy (Konjević \& Roberts 1976). In the analysis of the Kusch \& Oberschelp (1967) experiment, Konjević \& Roberts (1976) have found large variations of Stark widths within multiplets, and supposed that this may be caused by improper treatment of self-absorption. Moreover, Dimitrijević \& Konjević (1983) have shown on the basis of the analysis of Stark width systematic trends within spectral series, that the experimental results of Kusch \& Oberschelp (1967) are in disagreement with such trends.

Lakićević (1983) estimated on the basis of regularities and systematic trends Stark width and shift for the 
Zn I $4 \mathrm{~s}^{2}{ }^{1} \mathrm{~S}-4 \mathrm{p}^{1} \mathrm{P}^{\circ}$ transition for an electron temperature $(T)$ of $20000 \mathrm{~K}$ and an electron density of $10^{17} \mathrm{~cm}^{-3}$. He obtained the value of $0.066 \AA$ for the full width at half maximum, and $0.035 \AA$ for the shift. We obtain the value of $0.039 \AA$ for the width and $0.029 \AA$ for the shift. On the basis of regularities and systematic trends as well, Stark widths and shifts for the Zn I $4 \mathrm{p}^{3} \mathrm{P}^{\circ}-5 \mathrm{~s}^{3} \mathrm{~S}$ transition for electron temperatures of 10000 and $20000 \mathrm{~K}$ and an electron density of $10^{17} \mathrm{~cm}^{-3}$ have been estimated by Rathore et al. (1985). They obtained the value of $0.60 \AA$ for the full width at half maximum, and $0.36 \AA$ for the shift, for $T=10000 \mathrm{~K}$, and our results are $0.371 \AA$ for the width and $0.295 \AA$ for the shift. For $T=20000 \mathrm{~K}$, they obtained $0.61 \AA$ for the width and $0.30 \AA$ for the shift, and we $0.408 \AA$ for the width and $0.336 \AA$ for the shift. Particularly for the shift, this is in both cases an encouraging agreement of simple estimates with our semiclassical perturbation results.

The comparison between our semiclassical perturbation results and semiclassical results of Dimitrijević \& Konjević (1983) is shown in Table 4. Differences between the present calculations and the semiclassical method described in Griem (1974) and used by Dimitrijević \& Konjević (1983), have been discussed in detail in Dimitrijević \& Sahal-Bréchot (1995) and may be attributed to the theoretical differences and the differences in input data.

First of all, the lower cut-offs are different in both methods. In Dimitrijević \& Konjević (1983), the same cutoff for widths and shifts as well as for both elastic and inelastic collisions has been used. The effect of the change of the set of values for the cut-offs $R_{1}, R_{2}$, and $R_{3}$ has been studied and discussed in detail in Sahal-Bréchot (1969b): the final choice of the cut-offs was adopted for physical reasons (it allows for the unitarity of the S-matrix) and followed Seaton (1962).

Moreover, in the present method, with the help of the symmetrization procedure one takes into account the impact electron velocity change during an inelastic collision, which is not taken into account in Dimitrijević \& Konjević (1983). The importance of the symmetrisation has been shown in Sahal-Bréchot (1969b). It has been demonstrated there that the symmetrisation improves considerably (factor two) semiclassical cross sections for small energies (close to the threshold), which are overestimated without symmetrisation. Also, for the difference of the semiclassical method (Griem 1974) used by Dimitrijević \& Konjević (1983) we take into account explicitely the elastic collision contribution.

Another difference between methods used here and in Dimitrijević \& Konjević (1983), is the Debye shielding effect. Griem (1974) gives the equation for the Debye shielding correction, which may be included when necessary, and here, the Debye cut-off is included in the calculations. Also, while in Dimitrijević \& Konjević (1983) the
Table 4. Comparison between present Stark full widths at half maximum (WDSB) and shifts (dDSB) and the corresponding results (WDK, dDK) of Dimitrijević \& Konjević (1983)

\begin{tabular}{|c|c|c|c|c|c|c|}
\hline \multirow[t]{2}{*}{ Transition } & Wavel ength & $h T(K)$ & WDSB & $d D S B$ & WDK & $d D K$ \\
\hline & (A) & & (A) & (A) & (A) & (A) \\
\hline \multirow[t]{4}{*}{ Zn I $4 p-5 s$ (triplets) } & 4767.4 & 5000 & 0.320 & 0.243 & 0.408 & 0.195 \\
\hline & & 10000 & 0.371 & 0.295 & 0.432 & 0.226 \\
\hline & & 20000 & 0.408 & 0.336 & 0.502 & 0.251 \\
\hline & & 40000 & 0.443 & 0.337 & 0.596 & 0.253 \\
\hline \multirow[t]{4}{*}{$4 p-6 s$ (triplets) } & 3054.7 & 5000 & 0.676 & 0.449 & 0.812 & 0.367 \\
\hline & & 10000 & 0.752 & 0.565 & 0.920 & 0.404 \\
\hline & & 20000 & 0.809 & 0.562 & 1.122 & 0.420 \\
\hline & & 40000 & 0.889 & 0.521 & 1.338 & 0.396 \\
\hline \multirow[t]{4}{*}{$4 p-4 d$ (triplets) } & 3324.8 & 5000 & 0.423 & -0.107 & 0.520 & -0.137 \\
\hline & & 10000 & 0.488 & -0.0469 & 0.582 & -0.0995 \\
\hline & & 20000 & 0.543 & $0.714 E-04$ & 0.662 & -0.0548 \\
\hline & & 40000 & 0.583 & 0.0367 & 0.726 & -0.0163 \\
\hline \multirow[t]{4}{*}{$4 p-5 d$ (triplets) } & 2786.7 & 5000 & 2.33 & 0.481 & 3.96 & 0.364 \\
\hline & & 10000 & 2.77 & 0.509 & 4.04 & 0.288 \\
\hline & & 20000 & 3.08 & 0.458 & 4.08 & 0.230 \\
\hline & & 40000 & 3.21 & 0.410 & 3.96 & 0.191 \\
\hline
\end{tabular}

ion-broadening contribution is only a correction within the quasistatic theory, the complete semiclassical perturbation calculation has been performed here for the ion-impact broadening, when the impact approximation is valid. The differences in the imput data are the more recent atomic energy level data (Sugar \& Musgrove 1995).

Both methods have been compared with critically selected experimental data for 13 He I multiplets (Dimitrijević \& Sahal-Bréchot 1985) and it was found that the agreement between experimental data and both semiclassical methods is within the limits of 20 percent, which is the predicted accuracy of the semiclassical method (Griem 1974). One can see from Table 4 that for zinc differences are larger and increase with temperature, particularly for the shift. One must take into account that more recent and more complete energy levels have been used in our calculations.

The obtained Stark broadening data are of interest for a number of problems in astrophysics and plasma physics as e.g. abundance determinations, stellar spectra analysis and laboratory plasma diagnostics. Reliable experimental determinations of neutral zinc Stark broadening parameters will be of interest for checking and development of Stark broadening theory.

Acknowledgements. This work has been supported by the Ministry of Science and Technology of Serbia through the project "Astrometrical, Astrodynamical and Astrophysical Researches".

\section{References}

Barnard A.J., Cooper J., Smith E.W., 1974, J. Quant. Spectrosc. Radiat. Transfer 14, 1025 
Bates D.R., Damgaard A., 1949, Trans. Roy. Soc. London, Ser. A 242, 101

Biémont E., Godefroid M., 1980, A\&A 84, 361

Dimitrijević M.S., 1996, Zh. Prikl. Spektrosk. 63, 810

Dimitrijević M.S., 1997, Astrophys. Space Sci. 252, 415, Spektrosk. 63, 810

Dimitrijević M.S., Konjević N., 1983, J. Quant. Spectrosc. Radiat. Transfer 30, 45

Dimitrijević M.S., Sahal-Bréchot S., 1984, J. Quant. Spectrosc. Radiat. Transfer 31, 301

Dimitrijević M.S., Sahal-Bréchot S., 1985, Phys. Rev. A 31, 316

Dimitrijević M.S., Sahal-Bréchot S., 1995, Phys. Scr. 52, 42

Dimitrijević M.S., Sahal-Bréchot S., 1996, Phys. Scr. 54, 50

Dimitrijević M.S., Sahal-Bréchot S., Bommier V., 1991, A\&AS 89,581

Fishman I.S., Semin P.S., Desyatnik G.A., 1979, Opt. Spectrosc. 47, 245

Fleurier C., Sahal-Bréchot S., Chapelle J., 1977, J. Quant. Spectrosc. Radiat. Transfer 17, 595

Grechikhin L.I., 1969, J. Appl. Spectrosc. (USSR) 11, 870

Grevesse N., 1984, Phys. Scr. T8, 49

Griem H.R., 1974, Spectral Line Broadening by Plasmas. Academic Press, New York
Konjević N., Dimitrijević M.S., Wiese W.L., 1984, J. Phys. Chem. Ref. Data 13, 619

Konjević N., Roberts J.R., 1976, J. Phys. Chem. Ref. Data 5, 209

Konjević N., Wiese W.L., 1990 J. Phys. Chem. Ref. Data 19, 1307

Kusch H.J., Oberschelp E., 1967, Z. Astrophys. 67, 77

Lakićević I.S., 1983, A\&A 127, 37

Oertel G.K., Shomo L.P., 1968, ApJS 16, 175

Rao P.M.R., Saraswathy P., Krishnamarty G., et al., 1989, Pramana 32, 627

Rathore B.A., Purić J., Ćuk M., Lakićević I.S., 1985, Sing. J. Phys. 2, 105

Sahal-Bréchot S., 1969a, A\&A 1, 91

Sahal-Bréchot S., 1969b, A\&A 2, 322

Sahal-Bréchot S., 1974, A\&A 35, 321

Sahal-Bréchot S., 1991, A\&A 245, 322

Salakhov M.H., 1975, Dep. VINITI 2070-75

Seaton M.J., 1962, Proc. Phys. Soc. 79, 1105

Sneden C., Gratton R.G., Crocker D.A., 1991, A\&A 246, 354

Sugar J., Musgrove A., 1995, J. Phys. Chem. Ref. Data 24, 1803

Van Regemorter H., Hoang Binh Dy, Prud'homme M., 1979, J. Phys. B 12, 1073 